\title{
Capillary-gravity waves in a surface tension gradient. I: Discontinuous change
}

\author{
Shiyuan Gou \\ Department of Mechanical Engineering and Applied Mechanics, Universily of Michigan, \\ Ann Arbor, Michigan 48109 \\ Arthur F. Messiter \\ Department of Aerospace Engineering, University of Michigan, Ann Arbor, Michigan 48109 \\ William W. Schultz \\ Department of Mechanical Engineering and Applied Mechanics, University of Michigan, \\ Ann Arbor, Michigan 48109
}

(Received 14 July 1992; accepted 28 October 1992)

\begin{abstract}
When waves enter a region covered with a surfactant, the spatial variation of the surface tension causes reflection and refraction of the waves. The simplified inviscid-flow problem of two adjacent half-spaces with different surface tension coefficients is solved analytically for plane waves at oblique incidence. The linearized wave potential is described by a complex contour integral representation for which uniqueness is obtained from the requirement of energy conservation. The reflection is found to be small for some typical cases.
\end{abstract}

\section{INTRODUCTION}

Propagation of capillary waves in deep water has long been known ${ }^{1}$ to be influenced by the presence of a surface contaminant, through added viscous damping that results from the presence of the surfactant. In regions of the free surface covered with surfactant, the surface tension coeffcient is different from that in clean water; a gradient in surface tension is accompanied by a balancing viscous shear stress and therefore increased dissipation. Moreover, a wave encountering a gradient in surfactant concentration is expected to be partially reflected. For these reasons, surfactant slicks behind moving ships have sometimes been thought to be responsible for the dark return observed in synthetic aperture radar sensing of ship wakes. ${ }^{2}$ Here we seek to determine whether significant reflection of energy is possible as small-amplitude waves enter a region covered with surfactant.

To focus on calculating the reflection of capillarygravity waves, we neglect viscous effects and with this simplification consider a prescribed surfactant concentration in a vcry thin layer. For one limiting case, we assume that the surface tension coefficient has only two values, each occupying a half-plane, and that the change from one to the other occurs over a distance much smaller than a wavelength. Then the linear inviscid problem is further simplified to one with an abrupt change in surface tension coefficient across a straight line. The other limiting case, for a slow spatial variation in concentration, is considered in Part II.

Experiments of Sellin, ${ }^{3}$ Scott, ${ }^{4}$ and others have shown that a steady state with essentially discontinuous surface tension is possible, but that a small current is required to maintain this equilibrium. If the current is decreased, ${ }^{3}$ the surfactant begins to spread over the surface. Thus it is expected that a small current may be required for a fully self-consistent problem formulation, or at least that the time scale of the surface motion should be large in com- parison with one period of the wave motion. We omit these considerations here in the belief that useful information can be obtained without including additional effects, that the influence of other features may be secondary, and that refinements can be added in a later study.

For a plane wave at oblique incidence, the reflected and transmitted waves will likewise be plane at large enough distances from the discontinuity, with amplitudes that are consistent with energy conservation. But an additional condition is needed to complete the determination of the amplitudes, and this can be found only through a solution of a quite complicated local problem that provides flow details in a region close to the jump. In a related example, Hocking ${ }^{5}$ has given solutions for the reflection of a capillary-gravity wave moving obliquely toward a rigid vertical boundary, in which case the reflection depends on the behavior of the contact angle.

Surface wave problems with different boundary conditions on semi-infinite half-spaces have been studied previously. The two-dimensional dock problem (gravity waves encountering an inextensible surface) was first solved by Friedrichs and Lewy ${ }^{6}$ using an integral representation. The three-dimensional dock problem (oblique waves) was solved by Heins ${ }^{7}$ using a Wiener-Hopf method and by Stoker ${ }^{8}$ using methods similar to those of Friedrichs and Lewy. ${ }^{6}$ In the following we adopt the method of Friedrichs and Lewy ${ }^{6}$ and of Stoker ${ }^{8}$ and introduce an integral representation for the velocity potential. The solution is recognized as having a "local" part that disappears at large distances and a part consisting of plane traveling waves. An energy balance then leads to relationships among the amplitudes of these waves.

\section{ANALYSIS}

\section{A. Integral representation}

The flow is incompressible and inviscid, and so the potential function $\phi$ satisfies Laplace's equation. We seek 
solutions periodic in time with a single frequency that in dimensionless form is considered unity (along with the fluid density and gravitational acceleration). The two regions $x<0$ and $x>0$ have different dimensionless surface tension coefficients $T$ and $T^{+}$, respectively. The linearized free surface boundary conditions are determined in the usual way to give

$$
\phi_{t t}+\phi_{y}+T^{ \pm} \phi_{y y y}=0 \text { for } x \gtrless 0, \quad y=0 .
$$

Here $y$ points upward from the undisturbed free surface. The $z$ axis separates the two regions with different surface tension. In addition we require that $\nabla \phi \rightarrow 0$ as $y \rightarrow-\infty$ and at $x=+\infty$ there is an outgoing wave only.

As in the dock problem, we consider linear combinations of solutions having the form

$$
\operatorname{Re}\{f(x, y)\}\left\{\begin{array}{l}
\sin (m z-t) \\
\cos (m z-t)
\end{array}\right\},
$$

which satisfy the Laplace equation. Here $m$ is the wavenumber component in the $z$ direction and the wave is stationary in $x$; an oblique traveling wave can be constructed from two linearly independent solutions of the form (2). The Laplace equation becomes a Helmholtz equation for the complex amplitude $f(x, y)$, which is

$$
f_{x x}+f_{y y}-m^{2} f=0 \text {. }
$$

Following Stoker, ${ }^{8}$ we write the function $f(x, y)$ in the integral form

$$
f(x, y)=\frac{1}{2 \pi} \int_{P} F(\zeta) \exp \left(\zeta w+\frac{m^{2}}{4 \zeta} w^{*}\right) d \zeta
$$

Here $w=x+i y$, the asterisk $(*)$ indicates a complex conjugate, and $\zeta$ is a dummy variable of integration. The radiation conditions at $x= \pm \infty$ are replaced by finiteness conditions because the waves in (2) are stationary in $x$. The exponential term in (4) represents waves with varying wave number and satisfies the Helmholtz equation (as found by separation of variables). The complete solution is a linear combination of all these waves, with the amplitude $F$ chosen to satisfy the boundary conditions. As in Friedrichs and Lewy, ${ }^{6}$ the path of integration $P$ runs from 0 to $-i \infty$. The surface boundary condition (1) at $y=0$ becomes

$$
\operatorname{Re}\left\{f(x, 0)-f_{y}(x, 0)-T^{ \pm} f_{y y y}(x, 0)\right\}=0 \text { for } \quad x \gtrless 0 .
$$

Substituting (4) into (5) gives

$$
\begin{aligned}
& \operatorname{Im}\left\{\int_{P}\left[T^{ \pm} v^{3}(\xi)-v(\xi)-i\right] F(\xi) \exp \left(\xi x+\frac{m^{2}}{4 \xi} x\right) d \xi\right\} \\
& \quad=0 \text { for } x \geqslant 0,
\end{aligned}
$$

where

$$
v(\zeta)=\zeta-\frac{m^{2}}{4 \zeta}
$$

To achieve the proper behavior as $x \rightarrow \pm \infty$, we choose $F$ such that $\left(T^{+} v^{3}-v-i\right) F(\zeta)$ is analytic in the lower halfplane to the left of $P$ and $\left(T^{-} v^{3}-v-i\right) F(\zeta)$ is analytic in



FIG. 1. Roots of $T^{+} v^{3}-v-i=0$.

the lower half-plane to the right of $P$. Then we can swing the path of integration $P$ in the $\xi$ plane to the positive real axis when $x<0$ and to the negative real axis when $x>0$, because the integrand is analytic in $\zeta$ in the appropriate regions. Since the path of integration is now along the real axis, a real integrand will satisfy (6). Hence, we require

$$
\operatorname{Im}\left\{\left(T^{ \pm} v^{3}-v-i\right) F(\zeta)\right\}=0 \text { for } \zeta \$ 0 .
$$

From the above constructions, $F(\zeta)$ can have simple poles in the lower half-plane which are roots of $T^{ \pm} v^{3}-v-i=0$. We take $-\pi<\arg \zeta<\pi$. The six roots of $T^{+} v^{3}-v-i=0$ in the $\zeta$ plane are denoted by $\alpha_{k}$ and are shown in Fig. 1. The roots of $T^{-} v^{3}-v-i=0$ are similar and are denoted by $\beta_{k}$. We note that $\alpha_{1}, \alpha_{2}, \beta_{1}$, and $\beta_{2}$ are on the negative imaginary axis and are the only poles of $F(\zeta)$ in the lower half-plane. Here $\alpha_{1}$ and $\alpha_{2}$ correspond to the solution of $v(\zeta)=\zeta-\left(m^{2} / 4 \zeta\right)=-i l^{+}$with $l^{+}$the real wave number at $x=+\infty$ which satisfies the dispersion relation $T^{+}\left(l^{+}\right)^{3}+l^{+}-1=0$. Similarly, $\beta_{1}$ and $\beta_{2}$ correspond to a wave number $l^{-}$. The other roots can be found by factoring $v+i l^{\perp}$ from the corresponding equations. The roots can be shown to have the following important properties:

$$
\begin{aligned}
& \alpha_{1} \alpha_{2}=\alpha_{3} \alpha_{4}=\alpha_{5} \alpha_{6}=-m^{2} / 4, \\
& \alpha_{5}=-\alpha_{3}^{*} \quad \text { and } \quad \alpha_{6}=-\alpha_{4}^{*} .
\end{aligned}
$$

The roots $\beta_{k}$ satisfy similar relations. The integral path $P$ is chosen so that $\alpha_{1}$ and $\alpha_{2}$ lie to the left of $P$ and $\beta_{1}$ and $\beta_{2}$ lie to the right of $P$, as shown in Fig. 2. These poles are required for the oblique traveling waves at infinity.

\section{B. Determination of the amplitude function}

Given the imaginary part of an analytic function on the real axis, we can use Cauchy's formula to determine the complete function. The imaginary part of the logarithm of an analytic function is the phase, or argument, of the func- 


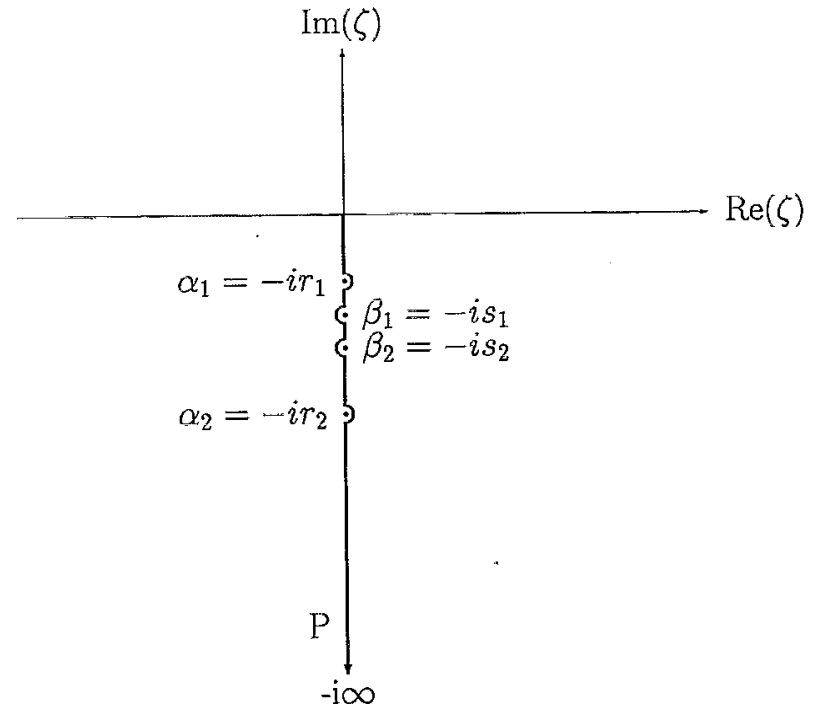

FIG. 2. Path of integration $P$.

tion. Since (8) gives the phase of $F$ on the real axis, we can introduce a suitable logarithm and then use Cauchy's formula.

If we further assume $F(\zeta) \neq 0$ on the positive and negative parts of the real axis, then the terms $\left(T^{ \pm} v^{3}-v-i\right) F(\zeta)$ do not change sign for $\zeta \lessgtr 0$, respectively. Without loss of generality we can assume that $\left(T^{-} v^{3}-v-i\right) F(\xi)>0$ for $\xi>0$. Now for $\zeta<0$, we can ensure $\zeta^{n}\left(T^{+} v^{3}-v-i\right) F(\zeta)>0$ by choosing an appropriate (even or odd) integer $n$. As will be seen later, the factor $\zeta^{n}$ also allows additional solutions which differ in their behavior at the origin. Then we have

$$
\xi^{n}\left(T^{ \pm} v^{3}-v-i\right) F(\zeta)>0 \text { for } \zeta \$ 0 .
$$

Introducing a new complex function,

$$
u(\zeta)=\log \left[\zeta^{n}\left(T^{-} v^{3}-v-i\right)\left(T^{+} v^{3}-v-i\right) F(\zeta)\right],
$$

results in an analytic $u(\zeta)$ in the lower half-plane. We will solve for $u$ and recover $F$ from the above equation. After applying the boundary conditions (11) we find the imaginary part of $u(\zeta)$ on the real axis as

$$
\begin{aligned}
u_{i}(\zeta) & =\operatorname{Im}\left\{\log \left(T^{ \pm} v^{3}-v+i\right)\right\} \\
& =\frac{1}{2 i} \log \left(\frac{T^{ \pm} v^{3}-v-i}{T^{ \pm} v^{3}-v+i}\right) \text { for } \zeta \gtrless 0 .
\end{aligned}
$$

The problem of solving the partial differential equation (3) with complex boundary conditions (5) is now converted into a simple boundary-value problem for an analytic function $u(\zeta)$.

Knowing $u_{i}(\zeta)$ and hence its derivative $u_{i}^{\prime}(\zeta)$ on the real axis, we can find $u^{\prime}(\zeta)$ in the following way when $\zeta$ is in the lower half-plane. Cauchy's integral formula gives

$$
u^{\prime}(\zeta)=\frac{1}{2 \pi i} \oint_{Q} \frac{u^{\prime}(\eta)}{\eta-\zeta} d \eta
$$

where the path of integration $Q$ consists of the real axis and a semicircle of large radius in the lower half-plane. If we assume $u^{\prime}(\zeta) \rightarrow 0$ as $|\zeta| \rightarrow \infty$ and let the radius of the semicircle be infinitely large, Eq. (14) becomes

$$
u^{\prime}(\zeta)=-\frac{1}{2 \pi i} \int_{-\infty}^{\infty} \frac{u^{\prime}(\eta)}{\eta-\zeta} d \eta
$$

The left-hand side of (15) becomes zero if we change $\zeta$ to $\xi^{*}$ which is outside of the integration contour. Taking the complex conjugate, we find

$$
i \int_{-\infty}^{\infty} \frac{u_{i}^{\prime}(\eta)}{\eta-\xi} d \eta=\int_{-\infty}^{\infty} \frac{u_{r}^{\prime}(\eta)}{\eta-\xi} d \eta
$$

where $u_{r}$ is the real part of $u(\xi)$. The result can also be obtained by considering solutions of the Laplace equation with boundary values prescribed along the axis. Substituting (16) into (15) we finally obtain

$$
u^{\prime}(\zeta)=-\frac{1}{\pi} \int_{-\infty}^{\infty} \frac{u_{i}^{\prime}(\eta)}{\eta-\zeta} d \eta
$$

Substituting the derivative of $u_{i}$ in (13) into this formula and integrating by parts results in

$$
u^{\prime}(\zeta)=u_{1}^{\prime}(\zeta)+u_{2}^{\prime}(\zeta)
$$

where

$$
\begin{aligned}
u_{1}^{\prime}= & \frac{1}{2 \pi i} \sum_{k=1}^{6}\left(\frac{1}{\zeta-\alpha_{k}} \log \frac{\zeta}{\alpha_{k}}-\frac{1}{\zeta-\alpha_{k}^{*}} \log \frac{\zeta}{\alpha_{k}^{*}}\right. \\
& \left.-\frac{1}{\zeta-\beta_{k}} \log \frac{\zeta}{\beta_{k}}+\frac{1}{\zeta-\beta_{k}^{*}} \log \frac{\zeta}{\beta_{k}^{*}}\right),
\end{aligned}
$$

and additional terms denoted by $u_{2}^{\prime}$ are needed since $u$ is analytic in the lower half-plane and the branches of the logarithms must be chosen for consistency with (13). Since (19) is real when $\zeta>0$, it follows from (13) that

$$
\operatorname{Im}\left\{u_{2}^{\prime}-\sum_{k=1}^{6} \frac{1}{\xi-a_{k}}\right\}=0
$$

for $\zeta>0$. Noting that $u_{2}^{\prime}=u^{\prime}-u_{1}^{\prime}$ must be analytic in the lower half-plane, we find, using (19) and (20),

$$
u_{2}^{\prime}=\sum_{k=3}^{6} \frac{1}{\zeta-\alpha_{k}}-\sum_{k=1}^{2} \frac{1}{\zeta-\alpha_{k}^{*}} .
$$

With arg $\zeta=-\pi$ for $\zeta<0$, it can be verified that the sum of (19) and (21) is also consistent with (13) on the negative real axis. It is easy to see that $u^{\prime}(\xi)=O(1 / \xi)$ as $\xi \rightarrow \infty$, so that the assumption for Eq. (15) is satisfied. With $u^{\prime}(\xi)$ determined in this way, we can find $F(\zeta)$ as

$$
F(\zeta)=\zeta^{-n} \frac{1}{T^{+} v^{3}-v-i} \frac{1}{T^{-} v^{3}-v-i} e^{u(\zeta)},
$$

where

$$
u(\zeta)=\int_{0}^{\zeta} u_{1}^{\prime}(\eta) d \eta+u_{2}(\xi)+\log C
$$




$$
u_{2}(\xi)=\log \frac{\left(\xi-\alpha_{3}\right)\left(\xi-\alpha_{4}\right)\left(\xi-\alpha_{5}\right)\left(\xi-\alpha_{6}\right)}{\left(\xi-\alpha_{1}^{*}\right)\left(\xi-\alpha_{2}^{*}\right)}
$$

and $C$ is a positive constant which will be chosen later. This $F(\zeta)$ satisfies all the assumptions. We can fix the lower limit of the above integral because this results in only a difference in $C$.

Now we can find $f(x, y)$ from (4) with $F(\zeta)$ given by (22). To see the properties of this solution more clearly, we swing the path $P$ onto the real axis. When $x<0$, we can change the path $P$ to the positive real axis $\xi>0$ by crossing poles at $\beta_{1}=-i s_{1}$ and $\beta_{2}=-i s_{2}$. Then, including contributions proportional to the residues of $F$ at these poles, and noting that $\beta_{1} \beta_{2}=-m^{2} / 4$, we obtain

$$
\begin{aligned}
f(x, y)= & \frac{1}{2 \pi} \int_{0}^{\infty} F(\zeta) \exp \left(\zeta w+\frac{m^{2}}{4 \xi} w^{*}\right) d \xi \\
& +i \exp \left[-i\left(s_{1} w-s_{2} w^{*}\right)\right] \operatorname{Res}\{F(\zeta)\}_{\zeta=\beta_{1}} \\
& +i \exp \left[-i\left(s_{2} w-s_{1} w^{*}\right)\right] \operatorname{Rcs}\{F(\zeta)\}_{\zeta=\beta_{2}} .
\end{aligned}
$$

It will be clear later that the integration along the real axis gives a local solution which decays to zero as the distance from the discontinuity at $x=0$ increases to infinity, while the second and the third terms give plane waves that satisfy the radiation boundary conditions. A result analogous to (25) is obtained for $x>0$.

\section{Local solution}

We denote the first term (the local solution) in (25) by $I_{n}(x, y)$. It is easier to observe the symmetry and convergence of the local solution in another form by first splitting $I_{n}$ into two parts, where for $x<0$,

$$
\begin{aligned}
I_{n}= & \frac{1}{2 \pi} \int_{0}^{m / 2} F(\zeta) \exp \left(\zeta w+\frac{m^{2}}{4 \zeta} w^{*}\right) d \zeta \\
& +\frac{1}{2 \pi} \int_{m / 2}^{\infty} F(\zeta) \exp \left(\zeta w+\frac{m^{2}}{4 \zeta} w^{*}\right) d \zeta .
\end{aligned}
$$

We will show that

$$
\frac{e^{u(\bar{\zeta})}}{\bar{\zeta}}=-\left(\frac{e^{u(\zeta)}}{\zeta}\right)^{*},
$$

where $\bar{\xi} \equiv m^{2} /\left(4 \zeta^{*}\right)$, which allows us to combine these two integrations into one. Writing $\zeta=r e^{i \theta}$ in polar coordinates gives $\bar{\xi}=\bar{r} e^{i \theta}$, where $\bar{r}=m^{2} /(4 r)$. We have

$$
u_{1}(\bar{\zeta})=e^{i \theta} \int_{0}^{\bar{r}} u_{1}^{\prime}\left(s e^{i \theta}\right) d s,
$$

or, setting $\bar{s}=m^{2} /(4 s)$, and renaming the integration variable,

$$
u_{1}(\bar{\zeta})=-e^{i \theta} \int_{\infty}^{r} u_{1}^{\prime}\left(\bar{s}^{i \theta}\right) \frac{m^{2}}{4 s^{2}} d s .
$$

But from (9), (10), and (19) it can be shown, after some algebra, that

$$
u_{1}^{\prime}(\bar{\zeta}) m^{2} /\left(4 \zeta^{* 2}\right)=-\left[u_{1}^{\prime}(\zeta)\right]^{*} .
$$

Then substitution in (29) gives $u_{1}(\infty)=0$ and

$$
u_{1}(\bar{\zeta})=u_{1}^{*}(\xi) \text {. }
$$

\section{Applying}

$$
\frac{e^{u_{2}(\zeta)}}{\zeta}=\frac{T^{+} v^{3}(\zeta)-v(\zeta)-i}{T^{+}\left[v^{2}(\zeta)+\left(l^{+}\right)^{2}\right]}
$$

and $v(\bar{\zeta})=-v^{*}(\zeta)$, we obtain

$$
\frac{e^{u_{2}(\bar{\zeta})}}{\bar{\zeta}}=-\left(\frac{e^{u_{2}(\zeta)}}{\zeta}\right)^{*} \text {. }
$$

Finally, with the help of (31), Eq. (27) is proved. Defining

$$
G(\zeta)=\zeta^{n-1} F(\zeta) \exp \left(\zeta w+\frac{m^{2}}{4 \zeta} w^{*}\right),
$$

changing variables as in (29), and then using (22), we can combine the two integrations in (26) in the form

$$
\begin{aligned}
I_{n}(x, y)= & \frac{1}{2 \pi} \int_{m / 2}^{\infty} \xi^{-1}\left[\xi^{2-n} G(\zeta)\right. \\
& \left.-\left(\frac{m^{2}}{4 \zeta}\right)^{2-n} G^{*}(\zeta)\right] d \zeta
\end{aligned}
$$

for $x<0$. From (22), it is clear that $F(\zeta)$ has no singularity in $(m / 2, \infty)$ which means that $I_{n}(x, y) \rightarrow 0$ as $x \rightarrow-\infty$. [While this could have been seen directly from (26), the new form (35) is needed below to show a symmetry property of $I_{n}$.] By a similar analysis it can be shown that for $x>0, I_{n}$ again has a form similar to (35) and also that $I_{n} \rightarrow 0$ as $x \rightarrow+\infty$. Thus $I_{n}$ is the local solution and the traveling waves at infinity come from the second and third terms in (25).

\section{Far field solution}

To find the relationship between the second and the third terms in $(25)$, we note that $\beta_{2}=\bar{\beta}_{1}$. If we write $e^{u\left(\beta_{1}\right) / \beta_{1}}$ in its polar form

$$
e^{u\left(\beta_{1}\right) / \beta_{1}}=a^{-} e^{i \theta^{-}},
$$

and remembering (27), we find

$$
e^{u\left(\beta_{2}\right)} / \beta_{2}=-a^{-} e^{-i \theta^{-}} .
$$

Evaluation of $a^{-}$requires identifying the real part of $u$. From the expressions (19) and (21) for $u_{1}^{\prime}$ and $u_{2}^{\prime}$, with properties of $\alpha_{k}$ and $\beta_{k}$ as given by (9) and (10) and Fig. 1 , after considerable algebra it is found that when $\zeta$ is on the negative imaginary axis,

$$
\begin{aligned}
\operatorname{Re}(u)= & \frac{1}{2} \sum_{k=3}^{6} \log \left[\left(\zeta-\alpha_{k}\right)\left(\zeta-\beta_{k}\right)\right] \\
& -\frac{1}{2} \sum_{k=1}^{2} \log \left[\left(\zeta-\alpha_{k}^{*}\right)\left(\zeta-\beta_{k}^{*}\right)\right],
\end{aligned}
$$

where the summations can be shown to be real, and finally that 


$$
a^{-}=C l^{-} \sqrt{\frac{c_{g}^{-}\left(T^{-}-T^{+}\right)}{T^{+} T^{-}\left[\left(l^{+}\right)^{2}-\left(l^{-}\right)^{2}\right]}} .
$$

Here $c_{g}=\left(3 T l^{2}+1\right) / 2$ is the nondimensional group velocity and $l$ is the wave number. From (22), we see

$$
\begin{aligned}
i \operatorname{Res}\{F(\zeta)\}_{\zeta=\beta_{1}}= & i \beta_{1}^{1-n}\left(\frac{e^{u(\zeta)}}{\zeta} \frac{1}{T^{+} v^{3}-v-i}\right)_{\zeta=\beta_{1}} \\
& \times \operatorname{Res}\left\{\frac{1}{T^{-} v^{3}-v-i}\right\}_{\zeta=\beta_{1}} \\
= & \frac{1}{\sqrt{c_{g}^{-}\left(l^{-}\right)^{2} k^{-}}} i^{n-3} s_{1}^{2-n}
\end{aligned}
$$

with $\beta_{1}=-i s_{1}$ ( $s_{1}$ is real). A similar expression is found for the residue at $\zeta=\beta_{2}$. The constant $C$ has been chosen for convenience as

$$
C=2 \sqrt{T^{+} T^{-}\left[\left(l^{-}\right)^{2}-\left(l^{+}\right)^{2}\right]\left(T^{+}-T^{-}\right)},
$$

and $k^{-}=s_{2}-s_{1}=\sqrt{\left(l^{-}\right)^{2}-m^{2}}$ is the $x$ component of the wave number; also $l^{-}=s_{2}+s_{1}$.

We denote the sum of the second and the third terms in Eq. $(25)$ by $J_{n}(x, y)$, where

$$
\begin{aligned}
J_{n}= & \frac{1}{\sqrt{c_{g}^{-}}\left(l^{-}\right)^{2} k^{-}} i^{n-3}\left(s_{1}^{2-n} e^{i\left(k^{-} x+\theta^{-}\right)}\right. \\
& \left.+s_{2}^{2-n} e^{-i\left(k^{-} x+\theta^{-}\right)}\right) e^{l^{-}, y}
\end{aligned}
$$

for $x<0$, and an analogous representation is obtained for $x>0$. The possible values for the integer $n$ are not determined yet. From (35) it can be shown that

$$
\left(\frac{m}{2}\right)^{q} \operatorname{Re}\left(I_{q+2}\right)=-\left(\frac{m}{2}\right)^{-q} \operatorname{Re}\left(I_{2-q}\right) .
$$

Hence $(m / 2)^{n-2} \operatorname{Re}\left(I_{n}\right)$ is antisymmetric about $n=2$, and the same is found to be true for $(m / 2)^{n-2} \operatorname{Re}\left(J_{n}\right)$. Thus we only need to consider solutions with $n<2$. We also note that, at $x=0, G(\xi)=O\left(\xi^{-5}\right)$ as $\xi \rightarrow \infty$, from (22) and (38). From this property we know that at the point $x=y$ $=0$, if $n=1, I_{1}(x, y)$ has finite third derivatives; if $n=0$, $I_{0}(x, y)$ has finite second derivatives; and if $n=-1$, $I_{-1}(x, y)$ has finite first derivatives. For $n<-1$, the elevation $\eta$, proportional to $\operatorname{Re}\left(f_{y}\right)$, is singular at $x=y=0$. If we require the elevation $\eta$ to be finite, our only solutions are those for $n=1,0$, and -1 .

When $n-1$,

$$
\operatorname{Re}\left(J_{1}\right)=-e^{l^{-} y} \frac{1}{\sqrt{c_{g}^{-}} l^{-} k^{-}} \cos \left(k^{-} x+\theta^{-}\right) \quad \text { as } x \rightarrow-\infty ;
$$

when $n=0$,

$$
\operatorname{Re}\left(J_{0}\right)=e^{l^{-} y} \frac{1}{\sqrt{c_{g}^{-}} l^{-}} \sin \left(k^{-} x+\theta^{-}\right) \quad \text { as } x \rightarrow-\infty ;
$$

when $n=-1$, we have

$$
\begin{gathered}
\operatorname{Re}\left(J_{-1}\right)=e^{l^{-} y} \frac{\left(l^{-}\right)^{2}-\left(3 m^{2} / 4\right)}{\sqrt{c_{g}^{-}} l^{-} k^{-}} \cos \left(k^{-} x+\theta^{-}\right) \\
\text {as } x \rightarrow-\infty .
\end{gathered}
$$

Similar results are obtained for $x \rightarrow+\infty$, with all superscripts changed from - to + .

\section{E. Wave reflection and transmission}

Given the incoming wave, a transmitted traveling wave is uniquely constructed if there are only two linearly independent stationary solutions. But now we have three linearly independent solutions for $n=-1,0,1$. We need one more constraint at $x=y=0$. This difficulty arises because we have allowed singular behavior at $x=y=0$ in the form of an energy sink or source. To make the solution unique, we assume that the energy is balanced such that the energy flux into the region from $x=-\infty$ equals the energy flux out of the region to $x= \pm \infty$. If we express the traveling waves at infinity in the form

$$
\phi=A_{T} e^{i^{+} y} \cos \left(k^{+} x+\theta^{+}+m z-t\right) \quad \text { as } x \rightarrow+\infty
$$

and

$$
\begin{aligned}
& \phi=A_{I} e^{l^{-} y} \cos \left(k^{-} x+\theta^{-}+m z-t\right)+A_{R} e^{l^{-} y} \\
& \times \cos \left(k^{-} x+\theta^{-}-m z+t\right) \text { as } x \rightarrow-\infty,
\end{aligned}
$$

then the energy balance requires

$$
c_{g}^{-}\left(A_{I}^{2}-A_{R}^{2}\right) k^{-}=c_{g}^{+} A_{T}^{2} k^{+} \text {. }
$$

Two conditions are needed for determination of the amplitude ratios $A_{R} / A_{I}$ and $A_{T} / A_{I}$. The energy balance (49) provides one of these conditions, and the second is obtained from the local solution. From (2), the solutions for $\phi$ have the form

$$
\begin{aligned}
\phi(x, y, z, l)= & \left(k^{+} / l^{+}\right) \psi^{c}(x, y) \cos (m z-t) \\
& -l^{+} \psi^{s}(x, y) \sin (m z-t),
\end{aligned}
$$

where the constant factors are included for convenience. The representations (47) and (48) show that $\psi^{c}$ and $\psi^{s}$ are proportional, respectively, to $\cos \left(k^{ \pm} x+\theta^{ \pm}\right)$and $\sin \left(k^{ \pm} x+\theta^{ \pm}\right)$as $x \rightarrow \pm \infty$. From (47) and (48), along with (44)-(46) and the energy balance (49), it is found that $\psi^{s}$ is proportional to the function $f$ with $n=0$, while $\psi^{c}$ is a linear combination of the solutions $f$ for $n=-1$ and $n=1$ :

$$
\psi^{s}=\operatorname{Re}\left\{f(x, y)_{n=0}\right\}
$$

and

$$
\psi^{c}=\operatorname{Re}\left\{f(x, y)_{n=-1}-\frac{3 m^{2}}{4} f(x, y)_{n-1}\right\},
$$

where a convenient normalization has been used, and that

$$
\frac{A_{T}}{A_{I}}=\frac{2 \sqrt{c_{g}^{-}}\left(l^{-} l^{+} k^{-}\right)}{\sqrt{c_{g}^{+}\left[\left(l^{-}\right)^{2} k^{+}+\left(l^{+}\right)^{2} k^{-}\right]}},
$$




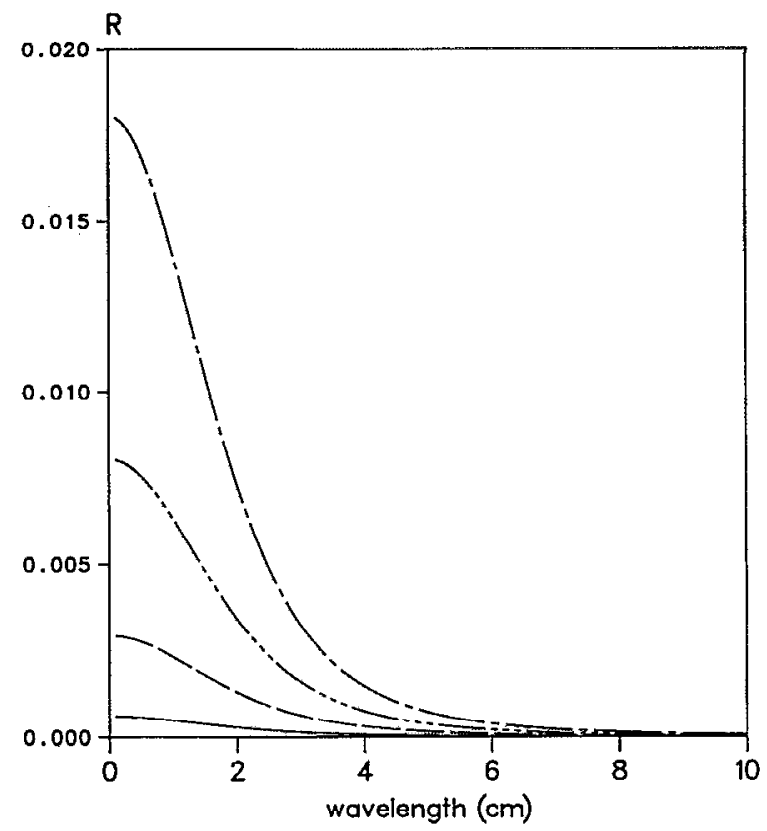

The phase shift $\theta^{+}-\theta^{-}$could also, in principle, be found from the local solution, but the calculation is more difficult and is not needed here.

The dispersion relation $T l^{3}+l=1$ shows that the wave number $l$ increases as the nondimensional surface tension coefficient $T$ decreases, and that the group velocity $c_{g}=\left(3 T l^{2}+1\right) / 2$ decreases as $l$ increases. (In the present notation, $l=1$ for pure gravity waves, $l=1 / 2$ at the minimum wave speed, and $l=0$ for pure capillary waves.) If the surface is clean for $x<0$ and contaminated for $x>0$, as in the numerical example discussed below, then $T^{+}<T^{-}$and $l^{+}>l^{-}$. Since $m$ has the same value for both $x<0$ and $x>0$, the wave-number vector is, of course, turned toward the $x$ axis if $l^{+}>l^{-}$(or $k^{+}>k^{-}$) and away from the $x$ axis if $l^{+}<l^{-}$(or $k^{+}<k^{-}$). While the energy flux of the transmitted wave is necessarily smaller than that of the incident wave, it follows from (53) that the amplitude of the transmitted wave can be larger than that of the incident wave when $T^{+}<T^{-}$, for certain ranges of the parameters. For example, at normal incidence it is found that $A_{T} / A_{I}>1$ if $k^{+}\left(1-2 k^{+} / 3\right) /\left(1+2 k^{+}\right)<k^{-}$.

The solutions also show rather curious behavior for the amplitude and phase of the reflected wave as the angle of incidence is changed. For normal incidence $m$ is equal to 0 and the amplitude of the reflected wave is always smaller than that of the incident wave, but the phase is the same when $T^{+}>T^{-}$and opposite when $T^{+}<T^{-}$. If $m$ is increased with $T^{+}$and $T^{-}$unchanged, the magnitude of the ratio $A_{R} / A_{I}$ decreases, and passes through zero when $m=l^{+} l^{-}\left[\left(l^{+}\right)^{2}+\left(l^{-}\right)^{2}\right]^{-1 / 2}$. For still larger $m$, there is a $180^{\circ}$ phase change from that at smaller $m$. The magnitude of the reflection coefficient approaches one as $m$ approaches the smaller of $l^{+}$and $l^{-}$,i.e., as the wave-number vector becomes more nearly parallel to the discontinuity on one side or the other.

For numerical examples illustrating the reflection of incoming wave energy from the discontinuity in surface tension coefficient, we take the density $\rho=10^{3} \mathrm{~kg} / \mathrm{m}^{3}$, with the surface tension coefficient $72 \mathrm{dyn} / \mathrm{cm}$ in clean water and $62,52,42$, and $32 \mathrm{dyn} / \mathrm{cm}$ in the contaminated region. The calculations were made for the case when the wave front is parallel to the $z$ axis, i.e., when $m=0$. Using these parameters, we can find the relationship between the energy reflection coeflicient $R=\left(A_{R} / A_{I}\right)^{2}$ and the wavelength $\lambda$ of the incident wave. These results in Fig. 3 show that the energy reflection is very weak. To see from the analysis why this is true, we can consider the behavior for small values of the jump $\Delta T=T^{+}-T^{-}$in the dimensionless surface tension coefficient, and therefore small values of the jump $\Delta k=k^{-}-k^{+}$in wave number. In this case $R$ is proportional to $[\Delta k /(2 k)]^{2}$, and $\Delta k / k$ is proportional to $\Delta T / T$ with a small numerical coefficient. The reflected energy in fact remains small even when $\Delta T / T$ is not small, and approaches zero as capillary effects decrease, i.e., when

FIG. 3. Reflection coefficient $R$ versus wavelength for normal incidence. Surface tension coefficient $T^{-}$is $72 \mathrm{dyn} / \mathrm{cm}$ in clean water for $x<0$ and $T^{+}$is $-: 62 ;--: 52 ;--_{-}-42 ;--.32 \mathrm{dyn} / \mathrm{cm}$ in contaminated water for $x>0$.

$\lambda$ becomes large in comparison with the value of $1.7 \mathrm{~cm}$ for minimum wave speed.

Using the same parameters as in Fig. 3 with fixed incident wavelength of $0.5 \mathrm{~cm}$, the relationship between $R$ and the angle of incidence is shown in Fig. 4. The ratio



FIG. 4. Reflection coefficient $R$ versus angle of incidence for incident wavelength $0.5 \mathrm{~cm}$. Surface tension coefficient is $72 \mathrm{dyn} / \mathrm{cm}$ in clean water for $x<0$ and $-: 62 ;--: 52 ;-\cdots---42 ;---: 32 \mathrm{dyn} / \mathrm{cm}$ in contaminated water for $x>0$. 


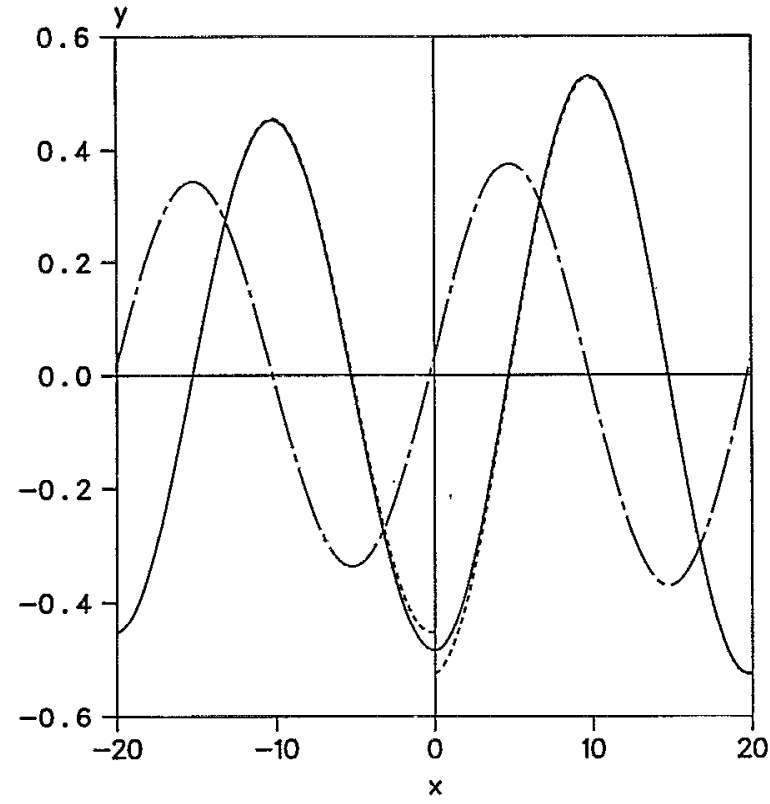

FIG. 5. Surface elevation corresponding to $\psi^{\mathrm{c}}(x, 0)$ :- $\longrightarrow$ : full solution; $\cdots$ : harmonic-wave solution valid for large $|x|$. Surface elevation corresponding to $\psi^{5}(x, 0)$ :- - - full solution and harmonic-wave solution. For both functions $T^{-} / T^{+}=2.25, m=l^{+} / 4$, wavelength in clean water for $x<0$ is $1 \mathrm{~cm}$, and the elevation is normalized as in (51) and (52).

$A_{R} / A_{I}$ is negative when the angle is zero and increases monotonically to one as the angle approaches $90^{\circ}$. There is an angle when the reflection is zero. The figure shows similar trends for different surface tension coefficients in the contaminated water.

The surface elevation corresponding to the functions $\psi^{c}(x, 0)$ and $\psi^{s}(x, 0)$ is plotted in Fig. 5, in nondimensional form for $m=l^{+} / 4$ and for a wavelength of $1 \mathrm{~cm}$ and a surface tension coefficient of $32 \mathrm{dyn} / \mathrm{cm}$ in the contaminated water, so that $T^{-} / T^{+}=2.25$. For given values of $z$ and $t$, the complete solution for the potential is a linear combination of these two functions. The numerical integration requires calculation of the function $f$ from (25) for $n=-1,0$, and 1 . This was accomplished using (42) for $J_{n}$ and (35) for $I_{n}$, in an alternate form with integration limits 0 and $m / 2$, along with the definitions (22) and (34) for $F$ and $G$, where $u$ is found from (19), (23), and (24).

Also shown in Fig. 5 are the harmonic-wave approximations to $\psi^{c}$ and $\psi^{s}$, correct only for large $|x|$ but plotted here all the way to $x=0$ in order to show more clearly the changes in $\psi^{c}$ and $\psi^{s}$ across the interaction region. The local solution $I_{n}$ contains a factor $C$, which is linear in $\Delta T$ for small $\Delta T$, so that $I_{n}$ approaches zero as $\Delta T$ approaches zero, whereas $J_{n}$ does not. Thus we can expect that the amplitude of the local solution is small compared to the amplitude of the harmonic waves. As $x$ increases from $-\infty$ to $+\infty$, it is seen from (47), (48), and (50) that the magnitude of $\left(k^{+} / l^{+}\right) \psi^{c}$ changes from $A_{I}+A_{R}$ to $A_{T}$ and the magnitude of $-l^{+} \psi^{s}$ changes from $A_{I}-A_{R}$ to $A_{T}$. For the example in Fig. 5, the change in $\psi^{s}$ is so small as to be imperceptible, and the change in $\psi^{c}$ is only a little larger than $10 \%$ of the amplitude. The local solution also is seen to have an appreciable value only within about one-half of a wavelength on either side of the discontinuity.

\section{CONCLUDING REMARKS}

Analytical solutions have been obtained, through a complex integral representation, to describe the reflection of a plane capillary-gravity wave encountering, at oblique incidence, an assumed discontinuity in surfactant concentration. At large distances from the discontinuity, the solution is given simply by incident, reflected, and transmitted plane waves. These waves alone, however, do not allow continuous surface elevation, and the integral representation provides continuity through an added local solution.

For a small jump in surface tension, the energy in the reflected wave is quadratic in the strength of the jump, with a small numerical coefficient. The reflection remains quite weak even for large changes in surface tension. The amplitude of the reflected wave is largest for a pure capillary wave and decreases toward zero as the wavelength increases. The solutions are derived for arbitrary angle of incidence and can be specialized to the case of normal incidence.

It is recognized that the approximation of inviscid flow should be augmented by a boundary-layer calculation that also allows the presence of a current. An extension of this kind is currently under study. It is believed, nevertheless, that the present formulation includes the effects that are dominant, and that additional features will provide a refinement without introducing large qualitative changes.

\section{ACKNOWLEDGMENTS}

This work was supported under the Program in Ship Hydrodynamics and the Program in Ocean Surface Processes and Remote Sensing at The University of Michigan, funded by The University Research Initiatives of the Office of Naval Research, Contracts No. N000184-86-K-0684 and No. N00014-92-J-1650.

${ }^{1} O$. Reynolds, "On the effect of oil on destroying waves on the surface of water," Br. Assoc. Rep. Papers 1, 409 (1880).

${ }^{2}$ R. D. Peltzer, J. H. Milgram, R. A. Skop, J. A. C. Kaiser, O. M. Griffin, and W. R. Barger, "Hydrodynamics of ship wake surfactant films," Proceedings of the 18th Symposium on Naval Hydrodynamics (National Academy, Washington, DC, 1990).

${ }^{3}$ R. H. J. Sellin, "Existence of a surface tension discontinuity at a liquid free surface," Nature 217, 536 (1968).

${ }^{4} J$. C. Scott, "Flow beneath a stagnant film on water: The Reynolds ridge," J. Fluid Mech. 116, 283 (1982).

${ }^{5}$ L. M. Hocking, "Capillary-gravity waves with boundaries: Threedimensional effects," Wave Motion 10, 301 (1988).

${ }^{6} \mathrm{~K}$. O. Friedrichs and H. Lewy, "The dock problem," Commun. Pure Appl. Math. 1, 135 (1948).

${ }^{7}$ A. E. Heins, "The scope and limitations of the method of Wiener and Hopf," Commun. Pure Appl. Math. 9, 447 (1956).

${ }^{8}$ J. J. Stoker, Water Waves (Interscience, New York, 1957), Chap. 5.4. 